\title{
Acoustic superradiance from an optical-superradiance-induced vortex in a Bose-Einstein condensate
}

\author{
Nader Ghazanfari* \\ Department of Physics, Mimar Sinan Fine Arts University, Bomonti 34380, Istanbul, Turkey \\ Özgür Esat Müstecaplığlu \\ Department of Physics, Koç University, 34450 Sariyer, Istanbul, Turkey \\ (Received 13 January 2014; revised manuscript received 10 March 2014; published 24 April 2014)
}

\begin{abstract}
We consider the scattering of an acoustic wave from a vortex induced by optical superradiance. The vortex is created by pumping a large amount of angular momentum with a Laguerre-Gaussian light beam in an atomic Bose-Einstein condensate. We derive the mean-field dynamical equations of the light-superfluid system, and obtain the equations governing the elementary excitation of the system, which result in a massless KleinGordon equation with source terms. This equation describes the propagation of the sound wave in an effective space-time. Employing a simplifying draining bathtub model for the vortex, we investigate the scattering of the acoustic wave in the vortex phase and obtain a condition for the acoustic superradiance. We conclude that Laguerre-Gaussian-beam-induced sudden transition from homogeneous to vortex state in the superfluid leads to a prominent observation of the acoustic superradiance.
\end{abstract}

DOI: 10.1103/PhysRevA.89.043619

PACS number(s): 03.75.Kk, 04.70.Dy, 04.80.-y

\section{INTRODUCTION}

The achievements in cooling and trapping of the ultracold dilute gases and developments in controlling their various properties makes it a favorite candidate for simulating different physical systems from solid-state to high-energy physics [1]. A fine control over dilute gases both experimentally and theoretically allows us, by analogy, to analyze the systems that are not easy to study, when dealing with the real one. In this paper, as an example of such efforts we theoretically investigate the possibility of the acoustic superradiance, the analog version of the Penrose process, which is the extracting of energy from the rotating black holes [2,3], mutually with the optical superradiance that happens in Bose-Einstein condensates.

Acoustic superradiance occurs in Bose-Einstein condensation when a sound wave scatters from a vortex with an effective curved space-time that is the geometry of rotating black holes. The event horizon in such a space-time exists inside a region called the ergoregion, and since the rotating energy of the black hole is located in between the event horizon and the ergosphere the extraction of energy becomes possible. In other words, in this process, the wave solution of the field equation is scattered from the ergoregion with an increase in its amplitude [4-7].

The theoretical framework to study the possible connection between motion of sound waves in a fluid flow and behavior of a quantum field in a classical gravitational field was constructed by Unruh in 1981. In his paper [8], Unruh showed that the equation describing the propagation of the acoustic fluctuation of the velocity potential in a barotropic, inviscid, and irrotational fluid is the same as the equation that governs the propagation of a massless scaler field in a curved space-time. Since that time the acoustic black holes have gathered a lot of attention. A relatively respectable amount of work [4-7,9-15] has been devoted to make analogies of different features of the black holes, among which spontaneous

\footnotetext{
*nghazanfari@msgsu.edu.tr
}

radiation [16] and stimulated emissions [2,3,17,18] are the most engaged properties.

On the other hand, an ensemble of atoms optically driven above a threshold intensity radiates in the form of superradiance [19,20]. The process occurs in Bose-Einstein condensates [21-25] for which above a threshold intensity the condensate undergoes another phase transition and rests in vortex state, in the case that the incident light carries angular momentum [26]. We aim to observe the acoustic aspect of superradiance along with optical superradiance in presence of an optically driven vortex. For this purpose, we consider a system of bosonic cold atoms cooled down to the condensate state in an elongated trap. The condensate is under a far off-resonant intense beam of laser (in our case Laguerre-Gaussian beams) pumped along the large axis of the trap. The light couples to the atoms and transfers angular momentum to the condensate. The large amount of angular momentum pumped to the condensate to create a vortex throughout a transition from normal state to optical superradiance state provides an opportunity to extract energy from this environment. This process eases the observation of the superradiance in an acoustic superradiance experiment.

The superradiance-induced vortex phase has been studied in detail in Ref. [26] and we work in this regime searching for the conditions of the acoustic superradiance for the system. Then, the equation of motion for acoustic fluctuation of the velocity field is derived from mean-field equations for the condensate order parameter and light modes. The resulting equation is a nonhomogeneous massless scalar field in an effective curved space-time. The possibility of observing superradiance for the system is discussed throughout the analytical method used in Refs. [4,5]. This paper is organized as follows: we describe the system writing the Hamiltonian in the first section giving the details of laser and also deriving the equations of motion for condensate and light modes order parameters. In addition we discuss the superradiance induced vortex state qualitatively. In the second section, we introduce the effective geometry of the acoustic black hole, writing the field equation for the 
phase perturbation of the condensate and discussing the metric and properties of rotating acoustic black hole. In Sec. III, we discuss the possibility of superradiance for the system by calculating the reflection coefficient throughout the scattering of a sound wave from a vortex. Finally we summarize the results in Sec. IV.

\section{SUPERRADIANCE-INDUCED VORTEX STATE}

We first review the main results and equations in Ref. [26], where the generation of a superradiance-induced vortex state by angular momentum carrying LG beam is studied. We consider a cigar-shaped Bose-Einstein condensate coupled to a far off-resonant intense laser field along the long axis of the trap. Atoms are interacting via short-range $s$-wave interaction. The many-body Hamiltonian describing the system is

$$
\begin{aligned}
H= & \int d^{3} \mathbf{r} \hat{\Psi}^{\dagger}(\mathbf{r}) H_{0} \hat{\Psi}(\mathbf{r})+\sum_{m} d^{3} \mathbf{k} \hbar \omega a_{k m}^{\dagger} a_{k m} \\
& +\sum_{m, m^{\prime}} \int d^{3} \mathbf{r} d^{3} \mathbf{k} d^{3} \mathbf{k}^{\prime} J\left(\mathbf{k}, \mathbf{k}^{\prime} ; \mathbf{r}\right) \hat{\Psi}^{\dagger}(\mathbf{r}) a_{k m}^{\dagger} a_{k^{\prime} m^{\prime}} \hat{\Psi}(\mathbf{r}) \\
& +\frac{1}{2} \int d^{3} \mathbf{r} d^{3} \mathbf{r}^{\prime} \hat{\Psi}^{\dagger}(\mathbf{r}) \hat{\Psi}^{\dagger}\left(\mathbf{r}^{\prime}\right) V\left(\mathbf{r}-\mathbf{r}^{\prime}\right) \hat{\Psi}(\mathbf{r}) \hat{\Psi}\left(\mathbf{r}^{\prime}\right),
\end{aligned}
$$

where $H_{0}$ is the atomic single-particle Hamiltonian consisting of a kinetic term and an external trapping potential, $V_{\text {ext }}(\mathbf{r})$, $\hat{\Psi}(\mathbf{r})$, and $a_{k m}$ are the annihilation operators for atoms and optical field, respectively, and $m$ and $m^{\prime}$ are labeling the angular momentum for optical modes. Here $V\left(\mathbf{r}-\mathbf{r}^{\prime}\right)=$ $4 \pi \hbar^{2} a_{s} / M \delta\left(\mathbf{r}-\mathbf{r}^{\prime}\right)$ is the two-body potential with $a_{s}$ being the $s$-wave scattering length and $M$ the mass of a single atom. The effective atom-light coupling coefficients $J\left(\mathbf{k}, \mathbf{k}^{\prime} ; \mathbf{r}\right)$ are given by

$$
J\left(\mathbf{k}, \mathbf{k}^{\prime} ; \mathbf{r}\right)=-\frac{\hbar J^{*}(\mathbf{k}) J\left(\mathbf{k}^{\prime}\right)}{\Delta} \Phi_{k m}^{*}(\mathbf{r}) \Phi_{k^{\prime} m^{\prime}}(\mathbf{r}),
$$

and determined by the single atom-photon dipole matrix element $g(\mathbf{k})$. Here, $\Delta$ is the detuning frequency, and $\Phi_{k m}(\mathbf{r})$ are the mode functions (in our case Laguerre-Gaussian modes) for the light field with the wave number $\mathbf{k}$. These mode functions are given as

$$
\begin{aligned}
\Phi_{k m}(\mathbf{r}) & =\phi_{m}(r) e^{i m \phi} e^{i k z} \\
& =\frac{1}{\sqrt{\pi}}\left(\frac{r}{a_{m}}\right)^{m} e^{-r^{2} / 2 a_{m}} e^{i m \phi} e^{i k z}
\end{aligned}
$$

The laser beam has a width of $a_{m}$ and carries $m \hbar$ units of orbital angular momentum. We write the Heisenberg equation of motion for four annihilation operators, and apply meanfield approximation, whereby the field operators are replaced by $c$ numbers. In particular, we replace $\hat{\Psi} \rightarrow \psi, a_{-k_{0} 1} \rightarrow$ $\alpha_{1}, a_{-k_{0} 0} \rightarrow \alpha_{2}, a_{k_{0} 0} \rightarrow \alpha_{3}$, and $a_{k_{0} 1} \rightarrow \alpha_{L}$, which lead us to expressions

$$
\begin{gathered}
i \partial_{t} \psi=\left[-\frac{\hbar}{2 M} \nabla^{2}+V+\frac{4 \pi a_{s} \hbar}{M}|\psi|^{2}-J_{l}\right] \psi \\
i \partial_{t} \alpha_{1}=\left[-\Delta_{1}-2 U_{0} I_{--}^{(11)}\right] \alpha_{1}-U_{0} I_{-+}^{(11)} \alpha_{L} \\
i \partial_{t} \alpha_{2}=\left[-\Delta_{2}-2 U_{0} I_{--}^{(00)}\right] \alpha_{2}-U_{0} I_{-+}^{(01)} \alpha_{L} \\
i \partial_{t} \alpha_{3}=\left[-\Delta_{3}-2 U_{0} I_{++}^{(00)}\right] \alpha_{3}-U_{0} I_{++}^{(01)} \alpha_{L},
\end{gathered}
$$

where $U_{0}=J_{k}^{2} / \Delta, \Delta_{i}$ 's are the end-fire mode frequencies in the rotating frame at frequency $\omega_{0}, J_{l}$ is the light-atom coupling

$$
\begin{aligned}
J_{l}= & 2 U_{0}\left\{\left|\alpha_{L}\right|^{2}\left|\Phi_{k_{0} 1}\right|^{2}+\left|\alpha_{1}\right|^{2}\left|\Phi_{-k_{0} 1}\right|^{2}\right. \\
& \left.+\left|\alpha_{2}\right|^{2}\left|\Phi_{-k_{0} 0}\right|^{2}+\left|\alpha_{3}\right|^{2}\left|\Phi_{k_{0} 0}\right|^{2}\right\} \\
& +U_{0}\left\{\alpha_{L} \alpha_{1}^{*} \Phi_{-k_{0} 1}^{*} \Phi_{k_{0} 1}+\alpha_{2}^{*} \Phi_{-k_{0} 0}^{*} \Phi_{k_{0} 1}\right. \\
& \left.+\alpha_{3}^{*} \Phi_{k_{0} 0}^{*} \Phi_{k_{0} 1}+\text { c.c. }\right\},
\end{aligned}
$$

and

$$
I_{\sigma \gamma}^{m m^{\prime}}=\int d^{3} \mathbf{r} \Phi_{\sigma k_{0} m}^{*}(\mathbf{r}) \Phi_{\gamma k_{0} m^{\prime}}(\mathbf{r})|\psi(\mathbf{r})|^{2} .
$$

Here $\sigma$, and $\gamma= \pm 1$ label the sign of the wave vectors with amplitude $k_{0}$.

Equation (4) is the Gross-Pitaevskii equation for a condensate coupled with a laser beam. Equations (4) and (5) have been solved numerically in Ref. [26] and the optical superradiance has been observed. In that paper, Tasgin et al. illustrate the dynamics of the transition from a condensate at its nonrotating ground state to a normal superradiance and then a rotatory superradiance and finally a superradianceinduced vortex phase for the condensate. We will work in this phase where after a certain density of laser beam two transitions happen and the superradiance with a topological vortex coexist. According to the dynamics of the transition discussed in Ref. [26], the mean photon number in mode $\alpha_{1}$ decreases dramatically, $\alpha_{2}$ remains unchanged but very small, and only the $\alpha_{3}$ mode survives in this phase where it increases sharply when superradiance take place. Assuming that the system resides in this regime we aim to find the possibility of observing the acoustic superradiance along with the optical superradiance when a sound wave scatters from the vortex.

\section{ACOUSTIC BLACK HOLE: EFFECTIVE GEOMETRY}

In order to investigate the possibility of observing the acoustic superradiance we need to write the equation governing the propagation of the acoustic fluctuation of the velocity potential in the effective geometry created by the vortex. We start from the Gross-Pitaevskii Eq. (4) and express the condensate order parameter in terms of its amplitude and phase, i.e., $\psi(\mathbf{r}, t)=\sqrt{\rho(\mathbf{r}, t)} e^{i S(\mathbf{r}, t)}$, where $\rho=|\Psi|^{2}$. This leads us us to two equations for real and imaginary parts of Eq. (4) as

$$
\begin{aligned}
\partial_{t} \rho(\mathbf{r}, t)= & -\frac{\hbar}{M}\left[\nabla \rho \cdot \nabla S+\rho \nabla^{2} S\right] \\
\partial_{t} S(\mathbf{r}, t)= & \frac{\hbar}{2 M} \frac{1}{\sqrt{\rho}} \nabla^{2} \sqrt{\rho}-\frac{\hbar}{2 M}|\nabla S|^{2} \\
& -V_{\text {ext }}-\frac{4 \pi a_{s} \hbar}{M} \rho+J_{l} .
\end{aligned}
$$

By linearizing Eqs. (8)-(9) and (5) for density, phase, and the light-atom coupling around the background values $\rho_{0}, S_{0}$, and $J_{0}$ in the optical superradiance-induced vortex phase, as

$$
\rho=\rho_{0}+\rho_{1}, \quad S=S_{0}+S_{1}, \quad J_{l}=J_{0}+J_{1},
$$


where $J_{0}$, and $J_{1}$ are

$$
\begin{aligned}
J_{0}= & 2 U_{0}\left[\left|\alpha_{L}\right|^{2}\left|\Phi_{k_{0} 1}\right|^{2}+\left|\alpha_{3}\right|^{2}\left|\Phi_{k_{0} 0}\right|^{2}\right] \\
& +U_{0} \alpha_{L}\left[\alpha_{3} \Phi_{k_{0} 0} \Phi_{k_{0} 1}^{*}+\text { c.c. }\right] \\
J_{1}= & U_{0} \alpha_{L}\left[\delta \alpha_{1} \Phi_{-k_{0} 1} \Phi_{k_{0} 1}^{*}+\delta \alpha_{2} \Phi_{-k_{0} 0} \Phi_{k_{0} 1}^{*}\right. \\
& \left.+\delta \alpha_{3} \Phi_{k_{0} 0} \Phi_{k_{0} 1}^{*}+\text { c.c. }\right]
\end{aligned}
$$

we obtain

$$
\begin{aligned}
\partial_{t} \rho_{1} & =-\frac{\hbar}{M}\left[\nabla \cdot\left(\rho_{0} \nabla S_{1}\right)-\nabla \cdot\left(\rho_{1} \nabla S_{0}\right)\right], \\
\partial_{t} S_{1} & =-\frac{\hbar}{M} \nabla S_{0} \cdot \nabla S_{1}-\frac{4 \pi a \hbar}{M} \rho_{1}+J_{1} .
\end{aligned}
$$

It should be noted that in Eq. (12) we have neglected the quantum pressure term,

$$
\frac{\hbar^{2}}{2 M}\left(\frac{1}{2 \sqrt{\rho_{0}}} \nabla \frac{\rho_{1}}{\sqrt{\rho_{0}}}-\frac{\rho_{1}}{2 \rho_{0}^{3 / 2}} \nabla^{2} \sqrt{\rho_{0}}\right) .
$$

Equations (11) and (12) should be solved together with the linearized equations of the modes $\alpha_{1}, \alpha_{2}$, and $\alpha_{3}$.

$$
\begin{aligned}
i \partial_{t} \delta \alpha_{1} & =-\Delta_{1} \delta \alpha_{1}-2 \frac{U_{0}}{\hbar} I_{--}^{11} \delta \alpha_{1}, \\
i \partial_{t} \delta \alpha_{2} & =-\Delta_{2} \delta \alpha_{2}-2 \frac{U_{0}}{\hbar} I_{--}^{00} \delta \alpha_{2}, \\
i \partial_{t} \delta \alpha_{3} & =-\Delta_{3} \delta \alpha_{3}-2 \frac{U_{0}}{\hbar} I_{++}^{00} \delta \alpha_{3} .
\end{aligned}
$$

In order to write Eqs. (11) and (12) in a compact form we can use the definitions for the the background flow velocity $\mathbf{v}$, and the speed of sound $c$ in a condensate,

$$
\mathbf{v}=\frac{\hbar}{M} \nabla S_{0}, \quad c=\frac{\hbar}{M} \sqrt{4 \pi a_{s} \rho_{0}} .
$$

We assume that the background density $\rho_{0}$ is constant, thus the speed of sound. Now Eqs. (11) and (12) can be combined and rewritten in a single equation for sound waves as

$$
\frac{1}{\sqrt{-g}} \partial_{\mu}\left(\sqrt{-g} g^{\mu \nu} \partial_{\nu} S_{1}\right)=-\partial_{t} J_{1}-\nabla \cdot\left(\mathbf{v} J_{1}\right),
$$

where $\mu$ and $v=0,1,2$, and $g^{\mu \nu}$, the inverse metric tensor is obtained as

$$
g^{\mu \nu}=\frac{1}{c^{2}}\left(\begin{array}{cccc}
-1 & \vdots & -v_{r} & -\frac{v_{\theta}}{r} \\
\cdots & \cdot & \cdots \cdots & \cdots \\
-v_{r} & \vdots & c^{2}-v_{r}^{2} & -\frac{v_{r} v_{\theta}}{r} \\
-\frac{v_{\theta}}{r} & \vdots & -\frac{v_{r} v_{\theta}}{r} & \frac{c^{2}-v_{\theta}^{2}}{r^{2}}
\end{array}\right) .
$$

Equation (17) is a nonhomogeneous massless Klein-Gordon equation in curved space-time, for which $g=\operatorname{det}\left(g_{\mu \nu}\right)$ and the metric tensor in polar coordinates is defined as

$$
g_{\mu \nu}=\left(\begin{array}{cccc}
-\left(c^{2}-v^{2}\right) & \vdots & -v_{r} & -r v_{\theta} \\
\cdots \cdots & \cdot & \cdots & \cdots \\
-v_{r} & \vdots & 1 & 0 \\
-r v_{\theta} & \vdots & 0 & r^{2}
\end{array}\right)
$$

This metric governs the propagation of the fluctuations (sound waves) and depends on the velocity field, and speed of sound thus the density of the condensate. Even though the dynamics of the atomic Bose-Einstein condensates is driven from a nonrelativistic equation, the behavior of the sound waves is specified by a relativistic equation in a curved spacetime $[8,10]$. The homogeneous form of the Eq. (17) introduced by Unruh [8] for a barotropic, inviscid, and irrotational fluid establishes the connection between the propagation of the scalar field in classical gravitational field and the wave sounds in curved space-time. We will discuss later, but it is worth to note, that the optical superradiance does not affect the effective curved space-time.

To observe the certain properties of the space-time it is better to write the metric from the metric tensor

$$
\begin{aligned}
d s^{2} & =g_{\mu \nu} d x^{\mu} d x^{\nu} \\
& =\left(v^{2}-c^{2}\right) d t^{2}-2 v_{r} d r d t-2 r v_{\theta} d \theta d t+d r^{2}+r^{2} d \theta^{2}
\end{aligned}
$$

The ergoshpere radius can be easily found from this metric and it is exactly where the temporal component of metric, i.e., $g_{00}$ changes sign. However to find the event horizon one needs to apply a coordinate transformation of form

$$
\begin{gathered}
d t \longrightarrow d t-\frac{v_{r}}{c^{2}-v_{r}^{2}} d r, \\
d \theta \longrightarrow d \theta-\frac{v_{r} v_{\theta}}{r\left(c^{2}-v_{r}^{2}\right)} d r,
\end{gathered}
$$

which results in the metric

$$
\begin{aligned}
d s^{2}= & -\left(c^{2}-v^{2}\right) d t^{2}+\left(\frac{c^{2}}{c^{2}-v_{r}^{2}}\right) d r^{2} \\
& -2 r v_{\theta} d \theta d t+r^{2} d \theta^{2} .
\end{aligned}
$$

The metric in the new coordinates has an obvious singularity at radial component which gives the radius of event horizon.

Now we need to specify the form of the flow velocity. The spatial profile of the superradiance-generated vortex is numerically determined in Ref. [26]. For our analytical examination, we simply choose a draining bathtub profile, which is a typical description of rotating acoustic black holes. This model was first used in Ref. [10] for rotating acoustic black holes, which is a $(2+1)$-dimensional flow with a sink or source at the origin. We assume that the density and velocity have pure radial dependency. The continuity equation with irrotationality and incompressibility of the flow leads us to write the velocity field as

$$
\mathbf{v}=\frac{A}{r} \hat{e}_{r}+\frac{B}{r} \hat{e}_{\theta},
$$

where $A$, and $B$ are constants and can be defined in terms of the black hole properties. The field equations derived from conservation laws mentioned above also result in a positionindependent background density $\rho_{0}$ throughout the flow, which automatically gives the constant speed of sound according to Eq. (16). Having the velocity field defined by Eq. (23), it can be easily checked that the ergosphere and event horizon are 
formed at $r_{\mathrm{erg}}$, and $r_{h}$, respectively

$$
r_{\mathrm{erg}}=\frac{\sqrt{A^{2}+B^{2}}}{c}, \quad r_{h}=\frac{|A|}{c} .
$$

The sign of $A$ is of no importance in determining the ergoregion, but it makes a difference when dealing with the event horizon. For positive $A$ the past event horizon is defined, which means we work with an acoustic white hole, while for negative $A$ the future event horizon is defined this time, which means we work with an acoustic black hole. We choose $A=-a c$, and $B=a^{2} \Omega$ where $a$ is the radius of the event horizon, and $\Omega$ is the angular velocity of the rotating black hole [7]. We will see that the growth of the ergosphere with increasing angular velocity of black hole will increase the amount of acoustic superradiance from the vortex. Now, we write the Klein-Gordon equation introduced above on this background in the more explicit form of

$$
\begin{aligned}
& {\left[-\frac{1}{c^{2}} \partial_{t}^{2}+\frac{2 a}{r} \partial_{t} \partial_{r}-\frac{2 \Omega a^{2}}{c^{2} r^{2}} \partial_{t} \partial_{\theta}+\left(\frac{c^{2} r^{2}-\Omega^{2} a^{4}}{c^{2} r^{4}}\right) \partial_{\theta}^{2}\right.} \\
& \left.\quad+\left(1-\frac{a^{2}}{r^{2}}\right) \partial_{r}^{2}+\frac{2 a^{3} \Omega}{c r^{3}} \partial_{r} \partial_{\theta}+\frac{a^{2}+r^{2}}{r^{3}} \partial_{r}-\frac{2 a^{3} \Omega}{c r^{4}} \partial_{\theta}\right] \\
& \quad \times S_{1}(\mathbf{r}, t)=-\partial_{t} J_{1}(\mathbf{r}, t)-\nabla \cdot\left[\mathbf{v} J_{1}(\mathbf{r}, t)\right]
\end{aligned}
$$

The homogeneous version of the equation above has been solved analytically [4-6] and numerically [7] and the superradiance has been observed. Since the superradiance is the extraction of energy from vortex, the problem can be reduced to find the reflection and transmission coefficients and discuss the possibility of finding a reflection probability greater than unity. The analytical method with some transformations gives the result relatively easily, but the numerical solution is not as easy. The method developed in Ref. [27] reduces the Klein-Gordon equation to a set of first-order equations by defining two conjugate fields; however, the resulting set of equations itself requires many numerical calculations. The method has been implied in Refs. [7,28] and the superradiance state has been discussed in detail. In the case of our system the problem becomes even more difficult since the equation must be solved along with the linearized equations of motions for $\delta \alpha_{1}, \delta \alpha_{2}$, and $\delta \alpha_{3}$. However, considering the dynamics of the condensate throughout the optical superradiance, since the $\alpha_{1}$ and $\alpha_{2}$ modes nearly vanish in this phase, one can neglect the contribution from these modes. Therefore, the analytical calculations reduce to solving Eq. (17), where the source term is determined by Eq. (15).

\section{SUPERRADIANCE}

The scattering properties of a sound wave from a superradiance-induced vortex is described by analyzing the massless Klein-Gordon equation (25). We separate the phase fluctuations $S_{1}$ into its variables by substitution of

$$
S_{1}(t ; r, \theta)=R(r) S(t ; \theta)=R(r) e^{i(n \theta-\omega t)},
$$

which results in a nonhomogeneous second-order differential equation for the perturbed phase. Here $n$ is the azimuthal quantum number with respect to the axis of rotation, and $\omega$ is the sound wave frequency. We divide both sides of the resulting equation by factor $l=1-a^{2} / r^{2}$ to obtain more familiar form of

$$
\frac{d^{2} R(r)}{d r^{2}}+P(r) \frac{d R(r)}{d r}+Q(r) R(r)=G(t ; r, \theta),
$$

where

$$
\begin{aligned}
P(r)= & \frac{1}{c r\left(r^{2}-a^{2}\right)}\left[c\left(a^{2}+r^{2}\right)+2 i \omega a r^{2}-2 i n \Omega a^{3}\right], \\
Q(r)= & \frac{1}{c^{2} r^{2}\left(r^{2}-a^{2}\right)}\left[n^{2} \Omega^{2} a^{4}+\omega^{2} r^{4}\right. \\
& \left.-n^{2} c^{2} r^{2}-2 n \omega \Omega a^{2} r^{2}-2 i n c \Omega a^{3}\right] .
\end{aligned}
$$

The source term in Eq. (25) includes the time and spatial derivatives, where the time dependency of $J_{1}$ in optical superradiance state is governed by Eq. (15). We can write $J_{1}(\mathbf{r}, t)=\tilde{G}(t ; \theta) J(r)$, where $\tilde{G}(t ; \theta)$ has the simple time dependency of the form $e^{i \omega_{l} t}$, with $\omega_{l}=\Delta_{3}+2 U_{0} I_{++}^{00} / \hbar$. Now, the source term in Eq. (27) can be then conveniently expressed as

$$
\begin{aligned}
G(t ; r, \theta)= & \frac{-1}{l S(t ; \theta)}\left[\partial_{t} \tilde{G}(t ; \theta)+\frac{\Omega a^{2}}{r^{2}} \partial_{\theta} \tilde{G}(t ; \theta)\right. \\
& \left.-\tilde{G}(t ; \theta) \frac{c a}{r} \frac{d}{d r}\right] J(r) .
\end{aligned}
$$

At the end of the previous section we discussed the dynamics of the condensate throughout the optical superradiance and emphasized that $J_{1}$ is a very simple expression only carrying $\phi_{0}$, and $\phi_{1}$ modes of Laguerre-Gaussian beam since only $\alpha_{3}$ survives in this phase. Thus, the position derivatives of $J$ gives a simple expression,

$$
\begin{aligned}
\frac{d J(r)}{d r} & =\frac{d}{d r}\left[\phi_{0} \phi_{1}\right]=\frac{d}{d r}\left[\left(\frac{r}{a_{1}}\right) e^{\frac{-r^{2}}{2 a_{\mathrm{red}}^{2}}}\right] \\
& =\left(\frac{1}{r}-\frac{r}{a_{\mathrm{red}}^{2}}\right) J(r),
\end{aligned}
$$

where $a_{\text {red }}^{2}=a_{0}^{2} a_{1}^{2} /\left(a_{0}^{2}+a_{1}^{2}\right)$ is the reduced width of the Laguerre-Gaussian beam.

Now we introduce a new coordinate $\tilde{r}$, known as the tortoise coordinate [29] and use the definition $d r=l d \tilde{r}$, which leads us to a transformation relation of

$$
\tilde{r}=r-\frac{a}{2} \ln \left|\frac{r+a}{r-c}\right| .
$$

Note that this transformation maps the horizon at $r_{h}=a$ to $\tilde{r} \longrightarrow-\infty$, and also maps $r \longrightarrow \infty$ to $\tilde{r} \longrightarrow \infty$. These mapping will be important when we check the behavior of the system at its asymptotic points. In order to investigate the possibility of the acoustic superradiance for our nonhomogeneous Klein-Gordon equation we follow a formal way used for a homogeneous one in Refs. [4-6], in which the superradiance is determined by the reflection and transmission coefficients. In order to facilitate the calculations of these coefficients we write the second-order differential equation (27) in the form of the usual Schrödinger equation. We set $R(r)=K(r) F(r)$, which along with the coordinate transformation give us,

$$
\frac{d^{2} F(\tilde{r})}{d \tilde{r}^{2}}+D(r) \frac{d F(\tilde{r})}{d \tilde{r}}+W(r) F(\tilde{r})=\frac{l^{2}}{K(r)} G(t ; r, \theta),
$$


where

$$
W(r)=\frac{l^{2}}{K(r)}\left[\frac{d^{2} K(r)}{d r^{2}}+P(r) \frac{d K(r)}{d r}+Q(r) K(r)\right] .
$$

Here $K(r)$ is obtained from the elimination of the first derivative term from differential equation Eq. (32), i.e., by equating $D(r)$ to zero,

$$
\frac{d K(r)}{d r}+\frac{1}{2}\left[P(r)+l \frac{d}{d r}\left(\frac{1}{l}\right)\right] K(r)=0 .
$$

By solving this equation for $K(r)$ and substituting $P(r)$ from definitions Eq. (28) one can obtain

$$
\begin{aligned}
K(r)= & \sqrt{r} \exp \left\{\left(\frac{i n a \Omega}{c}+1\right) \ln \left(\frac{1}{r}\right)\right. \\
& \left.-\frac{i a(\omega-n \Omega)}{2 c} \ln \left[c^{2}\left(r^{2}-a^{2}\right)\right]\right\} .
\end{aligned}
$$

We substitute $K(r)$ in Eq. (33) to obtain

$$
\begin{aligned}
W(r)= & \frac{1}{c^{2}}\left(\omega-\frac{n \Omega a^{2}}{r^{2}}\right)^{2}-\frac{1}{r^{2}}\left(n^{2}-\frac{1}{4}\right) \\
& +\frac{a^{2}}{r^{4}}\left(n^{2}-\frac{3}{2}\right)+\frac{5 a^{4}}{4 r^{6}} .
\end{aligned}
$$

Eventually, Eq. (32) becomes

$$
\frac{d^{2} F(\tilde{r})}{d \tilde{r}^{2}}+W(r) F(\tilde{r})=\frac{l^{2}}{K(r)} G(t ; r, \theta) .
$$

We scale the radial coordinate with length of the horizon, i.e., $r_{\text {new }}=r / a$, and the frequencies with sound wave frequency, $\omega_{\text {new }}=a \omega / c$, and $\Omega_{\text {new }}=a \Omega / c$. However to avoid using the new index we drop it and continue writing with old parameters.

In the asymptotic region when $r$, and $\tilde{r} \longrightarrow+\infty$ the terms with $1 / O(r)$ in $W(r)$ vanishes, and only the term with $\omega$ survives. The source term also vanished in this region due to the Gaussian term in $J(r)$. Thus, Eq. (37) becomes

$$
\frac{d^{2} F(\tilde{r})}{d \tilde{r}}+\omega^{2} F(\tilde{r})=0,
$$

which can be readily solved and written as a combination of incident wave and reflected one

$$
F(\tilde{r})=R e^{i \omega \tilde{r}}+e^{-i \omega \tilde{r}},
$$

so $R$ is the reflection coefficient. Now let us check the behavior of the differential equation around horizon when $r \longrightarrow 1$, and $\tilde{r} \longrightarrow-\infty$. In this region the nonhomogeneous term vanishes due to $l$, which is zero at horizon. Thus, Eq. (37) reduces to

$$
\frac{d^{2} F(\tilde{r})}{d \tilde{r}}+(\omega-n \Omega)^{2} F(\tilde{r})=0,
$$

for which the solution can be written in terms of transmission wave as

$$
F(\tilde{r})=T e^{i(\omega-n \Omega) \tilde{r}},
$$

where $T$ is the transmission coefficient. From the conservation law for current density we obtain the relation between reflection and transmission coefficients

$$
|R|^{2}=1+\left(\frac{n \Omega}{\omega}-1\right)|T|^{2},
$$

which leads us to the famous relation first obtained by Zeldovich [3] for the scattering of an electromagnetic wave with an orbital momentum $n$ and frequency $\omega$ from a cylinder rotating with an angular frequency $\Omega$. This relation indicates that for $\omega<n \Omega$, an amplifications occurs in reflection coefficient throughout the scattering, which is an evidence for the possibility of the acoustic superradiance in our analog system. Here $\Omega$ is related to the amount of angular momentum pumped to the condensate to create a superradiance-induced vortex state. Although Eq. (42) determines the region for superradiance it does not give the details of the scattering and its dependency on the sound wave frequency. Therefore we need a more detailed investigation of the reflection and transmission coefficients by solving the differential equation (27) explicitly. Previously in this section in order to get a condition for superradiance, we used the transformation $R(r)=K(r) F(r)$ on Eq. (27) and investigated the resulting equation in asymptotic limit where the source term has no effect. Similarly now we apply another transformation of the form $R(r)=r^{3 / 2} K(r) X(r)$ and applied the result of the asymptotic limit to find the reflection and transmission coefficients explicitly. Thus, Eq. (27) reduces to a homogeneous differential equation

$$
\begin{aligned}
& x(x+1) \frac{d^{2} X(x)}{d x^{2}}+(2 x+1) \frac{d X(x)}{d x} \\
& +\frac{1}{4}\left(\frac{u_{1}^{2}}{x}+\frac{1}{x+1}+1-u_{2}^{2}+\frac{a^{2} \omega^{2}}{c^{2}} x\right) X(x)=0,
\end{aligned}
$$

where

$$
u_{1}=\frac{a \omega}{c}\left(\frac{n \Omega}{\omega}-1\right), \quad u_{2}^{2}=n^{2}+\frac{2 a^{2} \omega^{2}}{c^{2}}\left(\frac{n \Omega}{\omega}-1\right) .
$$

Equation (43) is similar to the equation used by Starobinskii [17] to calculate the details of the amplification that occurs for the reflection coefficient during a superradiance from a rotating black hole. For sound waves with a wavelength $\lambda$ much larger than the radius of the horizon $a$, Eq. (43) reduces to the Riemann-Papparitz equation [31] with two regular singular point at $x=0,-1$, which has been investigated in details in Ref. [17] for rotating black holes and used in Ref. [30] for the acoustic superradiance from a vortex. Therefore, we assume that $a \ll \lambda$, which result in

$$
\begin{aligned}
& x(x+1) \frac{d^{2} X(x)}{d x^{2}}+(2 x+1) \frac{d X(x)}{d x} \\
& +\frac{1}{4}\left(\frac{u_{1}^{2}}{x}+\frac{1}{x+1}+1-u_{2}^{2}\right) X(x)=0 .
\end{aligned}
$$

The reflection coefficient can be calculated from this equation through the transformation of this differential equation to a hypergeometric form with known solutions [17,30]. The solutions near the horizon are the superposition of ingoing and outgoing parts, from which one can obtain the reflection and transmission coefficients

$$
|R|^{2}=1+\frac{2 a \omega}{c u_{2}\left|y_{1}-i y_{2}\right|^{2}}\left(\frac{n \Omega}{\omega}-1\right),
$$



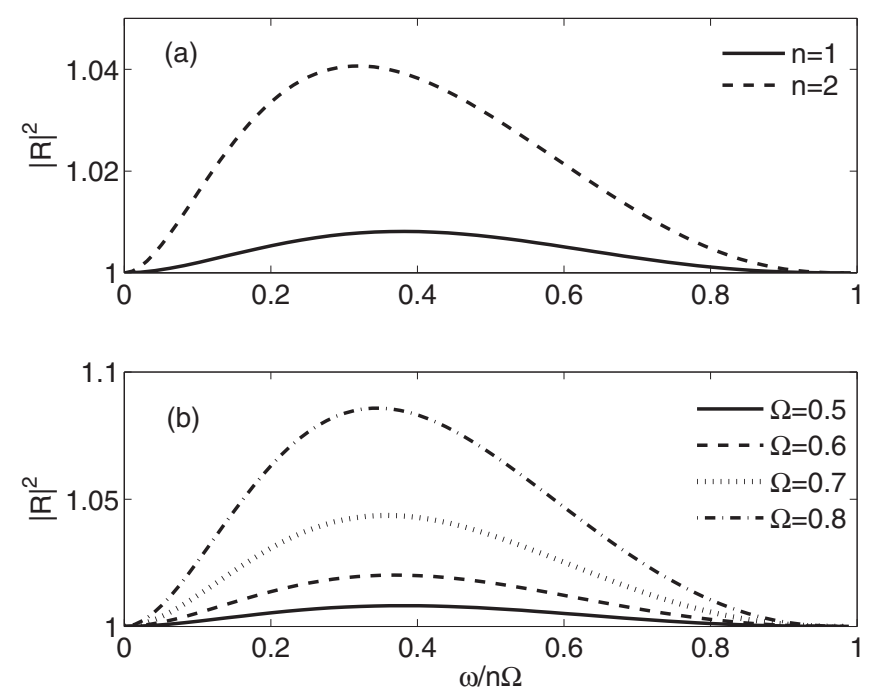

FIG. 1. The reflection amplitude $|R|^{2}$ as a function of $\omega / n \Omega$. (a) The reflection coefficient for $n=1,2$. The amplification in reflection coefficient increases by increasing the orbital angular momentum. (b) The reflection coefficient for different values of analog black hole angular frequency $\Omega$. The increase in reflection amplitude for large values of the angular frequency is more prominent than that for the large values of the orbital angular momentum.

where

$$
\begin{gathered}
y_{1}=\frac{\Gamma\left(1-i u_{1}\right) \Gamma\left(u_{2}\right)}{\Gamma\left(\frac{u_{2}}{2}-i \frac{u_{1}}{2}\right) \Gamma\left(1+\frac{u_{2}}{2}-i \frac{u_{1}}{2}\right)}, \\
y_{2}=\frac{\Gamma\left(1-i u_{1}\right) \Gamma\left(-u_{2}\right)}{\Gamma\left(\frac{-u_{2}}{2}-i \frac{u_{1}}{2}\right) \Gamma\left(1-\frac{u_{2}}{2}-i \frac{u_{1}}{2}\right)} .
\end{gathered}
$$

The transmission coefficient can be obtained easily from comparing Eq. (46) with Eq. (42). A detailed analysis of the reflection coefficient in Eq. (46) reveals the advantage of the investigation of the acoustic superradiance from an optical-superradiance-induced vortex. We demonstrate this in Fig. 1, which illustrates the amplification of reflection coefficient in Eq. (46) thorough the scattering of a sound wave from a vortex. Figure 1(a) compares the magnitude of the acoustic superradiance for two different modes, i.e., $n=1,2$, while Fig. 1(b) shows the magnitude of the superradiance for the first orbital angular momentum $n=1$ with different angular frequencies, i.e., $\Omega$ values. Comparing Figs. 1(a) and 1(b) exhibits that the superradiance for large values of angular frequency is more evident than the superradiance for the large values in orbital angular momentum. Since in our system the large amount of angular momentum is pumped to the condensate to obtain a optical superradiance-induced vortex the observation of acoustic superradiance throughout an experiment would be more prominent.

\section{SUMMARY AND DISCUSSION}

For a superradiance phase with an induced topological vortex in an atomic Bose-Einstein condensate we theoretically reveal the acoustic superradiance. This phenomenon is the analog of the Penrose process for rotating black holes [2,3]. The vortex state and superradiance phase are created by a sudden transfer of an incident angular momentum to the condensate [26]. In order to observe the optical superradiance mutually with the acoustic superradiance we assume that the condensate has gone through a phase transition to the optical superradiance induced vortex state. Since the optical superradiance phase happens with pumping a large amount of angular momentum around the vortex core, the extracting of energy from the ergoregion becomes easier. The effect of phase transition does not appear in the effective geometry of the vortex, but appears as a nonhomogeneous part in the Klein-Gordon equation describing the propagation of the sound wave in the introduced effective geometry, which is the geometry of a rotating black hole. The draining bathtub model fits the velocity field created by the optical superradiance. This model introduces an event horizon and an ergoregion. It is shown that the existence of the event horizon is not necessary to observe the Penrose process [6]. However, since the optical superradiance happens inside the event horizon the use of a fitting velocity field becomes essential.

The acoustic superradiance is determined for the vortex state as the amplification of the reflection coefficient, which becomes larger than unity [4-6] in this phase. We analytically show that the optical superradiance happens inside the effective event horizon and it does not affect the acoustic superradiance. The solutions of the nonhomogeneous Klein-Gordon equation in the asymptotic region gives the conservation law for the current density, which lead us to the acoustic superradiance mutually with optical superradiance. We obtain the same condition already introduced in Refs. [3-5]. We also calculate the reflection coefficient and show that the acoustic superradiance becomes more prominent for our system since there is a large amount of angular momentum in vortex state induced by optical superradiance. The acoustic superradiance condition exhibits that it happens for nonzero modes when the vortex angular frequency becomes larger than the sound wave propagation frequency. The full numerical solution of this problem would be illuminating to reveal the details of the superradiance transitions more explicitly.

\section{ACKNOWLEDGMENTS}

N.G. thanks TÜBITAK for the support.
[1] I. Bloch, J. Dalibard, and W. Zwerger, Rev. Mod. Phys. 80, 885 (2008).

[2] R. Penrose, Riv. Nuovo Cimento 1, 252 (1969); reprinted in Gen. Relat. Grav. 34, 1141 (2002).

[3] Ya. B. Zel'dovich, JETP Lett. 14, 180 (1971); Sov. Phys.-JETP 35, 1085 (1972).
[4] S. Basak and P. Majumdar, Classical Quant. Grav. 20, 3907 (2003).

[5] E. Berti, V. Cardoso, and J. P. S. Lemos, Phys. Rev. D 70, 124006 (2004).

[6] T. R. Slatyer and C. M. Savage, Classical Quant. Grav. 22, 3833 (2005). 
[7] F. Federici, C. Cherubini, S. Succi, and M. P. Tosi, Phys. Rev. A 73, 033604 (2006).

[8] W. G. Unruh, Phys. Rev. Lett. 46, 1351 (1981).

[9] W. G. Unruh, Phys. Rev. D 51, 2827 (1995).

[10] M. Visser, Classical Quant. Grav. 15, 1767 (1998).

[11] A. Retzker, J. I. Cirac, M. B. Plenio, and B. Reznik, Phys. Rev. Lett. 101, 110402 (2008).

[12] J. Macher and R. Parentani, Phys. Rev. A 80, 043601 (2009).

[13] M. Aspachs, G. Adesso, and I. Fuentes, Phys. Rev. Lett. 105, 151301 (2010).

[14] O. Lahav, A. Itah, A. Blumkin, C. Gordon, S. Rinott, A. Zayats, and J. Steinhauer, Phys. Rev. Lett. 105, 240401 (2010).

[15] Scott J. Robertson, J. Phys. B 45, 163001 (2012).

[16] S. W. Hawking, Nature (London) 248, 30 (1974); Commun. Math. Phys. 43, 199 (1975).

[17] A. Starobinski, Sov. Phys.-JETP 37, 28 (1973).

[18] B. DeWitt, Phys. Rep. 19, 295 (1975).

[19] R. H. Dicke, Phys. Rev. 93, 99 (1954).

[20] N. Skribanowitz et al., Phys. Rev. Lett. 30, 309 (1973).

[21] S. Inouye et al., Science 285, 571 (1999).
[22] D. Schneble, G. K. Campbell, E. W. Streed, M. Boyd, D. E. Pritchard, and W. Ketterle, Phys. Rev. A 69, 041601(R) (2004).

[23] L. Fallani, C. Fort, N. Piovella, M. Cola, F. S. Cataliotti, M. Inguscio, and R. Bonifacio, Phys. Rev. A 71, 033612 (2005).

[24] J. Li, X. Zhou, F. Yang, and X. Chen, Phys. Lett. A 372, 4750 (2008).

[25] L. Deng, M. G. Payne, and E. W. Hagley, Phys. Rev. Lett. 104, 050402 (2010).

[26] M. E. Taşgın, Ö. E. Müstecaplıŏglu, and L. You, Phys. Rev. A 84, 063628 (2011).

[27] M. A. Scheel, A. L. Erickcek, L. M. Burko, L. E. Kidder, H. P. Pfeiffer, and S. A. Teukolsky, Phys. Rev. D 69, 104006 (2004).

[28] C. Cherubini, F. Federici, S. Succi, and M. P. Tosi, Phys. Rev. D 72, 084016 (2005).

[29] R. M. Wald, General Relativity (University of Chicago Press, Chicago, 1984).

[30] S. Basak and P. Majumdar, Classical Quant. Grav. 20, 2929 (2003).

[31] M. Abramowitz and I. A. Stegun, Handbook of Mathematical Functions (Dover, New York, 1966). 